\title{
HOFER'S METRIC ON THE SPACE OF DIAMETERS
}

\author{
MICHAEL KHANEVSKY
}

\section{MAin RESUlT}

Let $D \subset \mathbb{R}^{2}$ be the open unit disk, endowed with the symplectic structure $\omega=\frac{1}{\pi} \mathrm{d} x \wedge \mathrm{d} y$ (so that $\left.\operatorname{Area}(D)=\int_{D} \omega=1\right)$. A diameter $L$ in $D$ is the image of a smooth curve $\gamma:(-1,1) \rightarrow D$ for which there exists $\varepsilon>0$ such that:

$$
\gamma(t)=(t, 0) \quad \forall t \in(-1,-1+\varepsilon] \cup[1-\varepsilon, 1),
$$

and which divides $D$ into two components of equal area. Denote by $\mathcal{E}$ the space of diameters in $D$, endowed with the Hofer metric $d$ (see the definition below). The present paper is dedicated to the study of metric properties of $(\mathcal{E}, d)$ and their relation to Lagrangian intersections.

Our first result is:

Theorem 1. The metric space $(\mathcal{E}, d)$ is unbounded: $\sup _{x, y} d(x, y)=\infty$.

In the context of Lagrangian intersections we prove:

Theorem 2. Let $L, L^{\prime} \subset D$ be two diameters intersecting transversely at \# $\left(L \cap L^{\prime}\right)$ points (see Section 3 for the precise definition). Then $d\left(L, L^{\prime}\right) \leq \frac{1}{8} \cdot \#\left(L \cap L^{\prime}\right)+1$. Moreover, this estimate is sharp in the sense that the linear bound cannot be improved. (However, the constant $\frac{1}{8}$ might not be the optimal one.)

The rest of the paper is organized as follows. In Section 2 we prove Theorem 1. Section 3 is devoted to the proof of Theorem 2, Finally, in Section 4 we discuss possible generalizations of our results to other surfaces than the disk.

Acknowledgements. I would like to thank prof. L. Polterovich for suggesting me the proof of Theorem 1 using the theory of Calabi quasimorphisms as well as for fruitful discussions. I also thank prof. P. Biran for his help with preparation of this paper.

Date: November 9, 2018.

The author was partially supported by the ISRAEL SCIENCE FOUNDATION (grant No. $1227 / 06^{*}$ ); This is a part of the author's $\mathrm{PhD}$ thesis, being carried out under the guidance of Prof. P. Biran, at Tel-Aviv University. 


\section{INFINITENESS OF THE DIAMETER}

The distance between two diameters $L_{1}, L_{2} \subset D$ is defined by

$$
d\left(L_{1}, L_{2}\right)=\inf _{\substack{\phi \in \operatorname{Ham}(D) \\ \phi\left(L_{1}\right)=L_{2}}}\|\phi\|
$$

where $\|\cdot\|$ stands for the Hofer norm on the group $\operatorname{Ham}(D)$ of compactly supported Hamiltonian diffeomorhisms:

$$
\|\phi\|=\inf \int_{0}^{1} \max _{p \in D} H(p, t)-\min _{p \in D} H(p, t) \mathrm{d} t,
$$

where the infimum goes over all compactly supported Hamiltonians $H: D \times[0,1] \rightarrow \mathbb{R}$ such that $\phi$ is the time- 1 map of the corresponding flow.

The distance $d$ is well defined as $\operatorname{Ham}(D)$ acts transitively on the space of diameters. Properties of Hofer metric on Ham imply that $d(\cdot, \cdot)$ is indeed a metric (for details, see [Ch]) and is invariant under Hamiltonian diffeomorphisms:

$$
d\left(L_{1}, L_{2}\right)=d\left(\phi L_{1}, \phi L_{2}\right), \quad \forall \phi \in \operatorname{Ham}(D) .
$$

Denote by $L_{0}=\{y=0\} \subset D$ the standard diameter. Denote by $S$ the stabilizer of $L_{0}$ in $\operatorname{Ham}(D)$, that is,

$$
S=\left\{\phi \in \operatorname{Ham}(D) \mid \phi\left(L_{0}\right)=L_{0}\right\},
$$

Transitivity of the action of $H a m$ on $\mathcal{E}$ implies the identification $\mathcal{E} \simeq$ $\operatorname{Ham}(D) / S$ with the left cosets of $S$. We define the reduced diameter space to be

$$
\hat{\mathcal{E}}=S \backslash \mathcal{E}=\{L \in \mathcal{E}\} / \sim,
$$

where $L \sim \phi L$ for any $\phi \in S$. An equivalent definition is

$$
\hat{\mathcal{E}}=" S \backslash \operatorname{Ham}(D) / S "=\{h \in \operatorname{Ham}(D)\} / \sim,
$$

with $h \sim \phi h \psi$ for any $\phi, \psi \in S$.

As $d\left(L_{0}, \phi L\right)=d\left(\phi L_{0}, \phi L\right)=d\left(L_{0}, L\right)$ for all $\phi \in S$, the distance from $L_{0}$ descends to $\hat{\mathcal{E}}$, hence is well defined on the reduced diameter space.

Theorem 1 follows from the following:

Proposition 3. $\sup _{L} d\left(L_{0},[L]\right)=\infty$, therefore the reduced space $\hat{\mathcal{E}}$ is unbounded.

In the proof we present an explicit autonomous Hamiltonian flow $\phi$ which deforms the standard diameter arbitrary far from $L_{0}$ as $t \rightarrow \infty$. 
The proof uses results from Entov-Polterovich E-P]. We start with a brief recollection of some relevant facts from that paper.

Let $F_{t}: D \rightarrow \mathbb{R}, t \in[0,1]$ be a time-dependent smooth function with compact support. We define $\widetilde{\operatorname{Cal}}\left(F_{t}\right)=\int_{0}^{1}\left(\int_{D} F_{t} \omega\right) \mathrm{d} t$. As $\omega$ is exact on $D, \widetilde{C a l}$ descends to a homomorphism $\operatorname{Cal}: \operatorname{Ham}(D) \rightarrow \mathbb{R}$ which is called the Calabi homomorphism. Clearly, for $\phi \in \operatorname{Ham}(D)$, $\operatorname{Cal}(\phi) \leq\|\phi\|$.

Let $G$ be a group. A function $r: G \rightarrow \mathbb{R}$ is called a quasimorphism if there exists a constant $R$ such that $|r(f g)-r(f)-r(g)|<R$ for all $f, g \in$ $G$. The quasimorphism $r$ is called homogeneous if it satisfies $r\left(g^{m}\right)=$ $m r(g)$ for all $g \in G$ and $m \in \mathbb{Z}$. Any homogeneous quasimorphism satisfies $r(f g)=r(f)+r(g)$ for commuting elements $f, g$.

For a compactly supported function $F: D \rightarrow \mathbb{R}$ the Reeb graph $T_{F}$ is defined as a set of connected componets of level sets of $F$ (for a detailed definition we refer the reader to $[\mathrm{E}-\mathrm{P}])$. For a generic Morse function $F$ this set, equipped with the topology induced by the projection $\pi_{F}$ : $D \rightarrow T_{F}$, is homeomorphic to a tree. We endow $T_{F}$ with a probability measure given by $\mu(A)=\int_{\pi_{F}^{-1}(A)} \omega$ for any $A \subseteq T_{F}$ with measureable $\pi_{F}^{-1}(A) \cdot \pi_{F}(\partial D)$ will be referred to as the root of $T_{F}$.

The results from E-P imply existance a family of homogeneous quasimorphisms $\left\{\mathrm{Cal}_{A}\right\}_{A \in[1 / 2,1)}$ on $\operatorname{Ham}(D)$ with the following properties:

- $\operatorname{Cal}_{A}(\phi)=\operatorname{Cal}(\phi)$ for any $\phi$ supported in a disk of area $A$.

- $\mathrm{Cal}_{A}(\phi) \leq 2 A \cdot\|\phi\|$.

- For $\phi \in \operatorname{Ham}(D)$ generated by an autonomous function $F$ : $D \rightarrow \mathbb{R}, \mathrm{Cal}_{A}(\phi)$ can be computed in the the following way. Consider the set $I$ of all points on the Reeb graph $T_{F}$ of $F$ which cut from the root subtrees of total measure at least $A$. $I$ is either a segment on $T_{F}$ which starts from the root or empty. For nonempty $I$ denote by $x$ the other end of the segment, for $I=\emptyset$ we set $x$ to be the root. Then $\operatorname{Cal}_{A}(\phi)=\operatorname{Cal}(\phi)-2 A \cdot F(x)$.

We now prove Proposition 3. Let

$$
r_{A}=\mathrm{Cal}_{A}-\mathrm{Cal}, \quad A \in[1 / 2,1) .
$$

For each $A, r_{A}$ is a homogeneous quasimorphism which is Lipschitz with respect to the Hofer distance on $\operatorname{Ham}(D)$ :

$$
\begin{aligned}
\left|r_{A}(\phi)\right| & =\left|\operatorname{Cal}_{A}(\phi)-\operatorname{Cal}(\phi)\right| \leq \\
& \leq 2 A \cdot\|\phi\|+\|\phi\| \leq(1+2 A)\|\phi\|
\end{aligned}
$$


We first show that $r_{A}$ vanishes on the stabilizer $S$. Let $\phi \in \operatorname{Ham}(M)$ be such that $\phi\left(L_{0}\right)=L_{0}$. Replacing $\phi$ with $\psi \circ \phi$ where $\psi \in \operatorname{Ham}(D)$ has arbitrary small norm we may assume that $\phi=$ Id in some neighborhood of $L_{0}$. From the transitivity of $\mathrm{Ham}$ on each half-disk it follows that $\phi$ can be decomposed as $\phi=\phi_{n} \circ \phi_{s}$ where $\phi_{n}\left(\phi_{s}\right)$ are Hamiltonians supported in the upper (lower) half of $D$ with respect to $L_{0}$. Since $\phi_{n}, \phi_{s}$ commute, we have

$$
\operatorname{Cal}(\phi)=\operatorname{Cal}\left(\phi_{n}\right)+\operatorname{Cal}\left(\phi_{s}\right)=\operatorname{Cal}_{A}\left(\phi_{n}\right)+\operatorname{Cal}_{A}\left(\phi_{s}\right)=\operatorname{Cal}_{A}(\phi)
$$

hence $r_{A}(\phi)=0$.

It follows that $r_{A}$ "descends" to $\hat{\mathcal{E}}$ in the following sense: for any $\phi \in \operatorname{Ham}(D), r_{A}(\phi)$ can be computed from $[\phi] \in \hat{\mathcal{E}}$ up to an error $C$ which is bounded by twice the defect of the quasimorphism. Moreover, we also have

$$
d\left(\phi\left(L_{0}\right), L_{0}\right) \geq \frac{\left|r_{A}(\phi)\right|-C}{1+2 A} .
$$

Assume that there exists a diffeomorphism $\phi \in \operatorname{Ham}(D)$ for which $r_{A}(\phi) \neq 0$ for some $A \in[1 / 2,1)$. Then

$$
d\left(\phi^{n}\left(L_{0}\right), L_{0}\right) \geq \frac{\left|r_{A}\left(\phi^{n}\right)\right|-C}{1+2 A}=\frac{n\left|r_{A}(\phi)\right|-C}{1+2 A} \underset{n \rightarrow \infty}{\longrightarrow} \infty
$$

Proposition 3 would follow if we show existence of such a $\phi$.

For this end, let $H_{\varepsilon}: D \rightarrow \mathbb{R}$ be an autonomous smooth function given by $H_{\varepsilon}(r, \theta)=-\frac{r^{2}}{2}+\frac{1}{2}$ on the disk $\{r \leq 1-\varepsilon\}$, and which equals zero near $\partial D$. Let $\phi$ be its time-1 map. Then the Reeb graph of $H_{\varepsilon}$ is a segment whose points correspond to circles around the origin (level sets of $\left.H_{\varepsilon}\right)$. The level set $x$ relevant for calculation of $\mathrm{Cal}_{A}(\phi)$ is the circle given by $r_{x}^{2}=A$ and so:

$$
\operatorname{Cal}_{A}(\phi)=\operatorname{Cal}(\phi)-2 A \cdot H_{\varepsilon}(x)=\operatorname{Cal}(\phi)+2 A \cdot \frac{A-1}{2} .
$$

It follows that

$$
r_{A}(\phi)=\operatorname{Cal}_{A}(\phi)-\operatorname{Cal}(\phi)=A(A-1) \neq 0 .
$$

This completes the proof of Proposition 3, hence of Theorem 1, too.

In the following section we prove that for any diameter $L$ which is transverse to $L_{0}, d\left(L_{0}, L\right) \leq K \cdot \#\left(L_{0} \cap L\right)+c$ (the proof is given for $\left.K=\frac{1}{8}\right)$.

At the same time, the quasimorphisms constructed above allow us to relate distance between diameters to the number of intersection points. 
We use the flow generated by $H_{\varepsilon}$ from the proof of Proposition 3 to obtain a lower bound for $K$.

Corollary 4. The optimal value for the coefficient $K$ is at least $\frac{1}{16}$.

Proof. Consider $\phi^{t}$ given by the time- $t$ map of the flow of $H_{\varepsilon}$ defined above. It rotates all points of the disk $\{r \leq 1-\varepsilon\}$ by angle $\pi t$, while each rotation by $\pi$ gives rise to two intersection points. For a careful choice of smoothing near the boundary (which doesn't generate any "unnecessary" intersections) we thus obtain $\#\left(L_{0} \cap \phi^{t} L_{0}\right) \leq 2 t+1$. Therefore

$$
\begin{aligned}
d\left(L_{0}, \phi^{t} L_{0}\right) & \geq \frac{\left|r_{A}\left(\phi^{t}\right)\right|-C}{1+2 A}=\frac{A(1-A) \cdot t-C}{1+2 A} \geq \\
& \geq \frac{A(1-A)}{2(1+2 A)}\left(\#\left(L_{0} \cap \phi^{t} L_{0}\right)-(2 C+1)\right) .
\end{aligned}
$$

Now note that the expression on the righthand side equals $\frac{\#\left(L_{0} \cap \phi^{t} L_{0}\right)}{16}+c$ for $A=1 / 2$.

\section{LINEAR BOUND}

Let $L^{\prime}, L^{\prime \prime} \subset D$ be two diameters. We say that they intersect transversely if the following holds: $\exists \varepsilon_{-1}, \varepsilon_{1}>0$ such that:

(1) $\left(-1,-1+\varepsilon_{-1}\right] \subset L^{\prime}, L^{\prime \prime}$

(2) $\left[1-\varepsilon_{1}, 1\right) \subset L^{\prime}, L^{\prime \prime}$

(3) $L^{\prime}$ intersects $L^{\prime \prime}$ transversely outside $\left(-1,-1+\varepsilon_{-1}\right] \cup\left[1-\varepsilon_{1}, 1\right)$ and the intesection set is compact.

If this is the case we denote by $\#\left(L^{\prime} \cap L^{\prime \prime}\right)$ the number of transverse intersection points between $L^{\prime}$ and $L^{\prime \prime}$. Obviously this number is finite. Note that a generic diameter satisfies the conditions of transverse intersection with $L_{0}$.

We now give a combinatorial description of the image of a diameter in the reduced space $\hat{\mathcal{E}}$.

Let $L=\phi\left(L_{0}\right) \subset D$ be a diameter intersecting $L_{0}$ transversely. We call a point $p \in D \backslash\left(L_{0} \cup L\right)$ northern (southern) if it belongs to the upper (lower) half of $D \backslash L_{0}$ and white (black) if it is an image of a northern (southern) point under $\phi$. We construct a graph $G(L)=(V, E)$ (see Figure 11) associated to $L$ as follows: the vertices $V=V(G)$ are the set of connected components of $D \backslash\left(L_{0} \cup L\right)$, and two vertices $v_{1}, v_{2} \in V$ are adjacent if they have a common boundary along a segment of $L_{0}$. Here we omit the two segments adjacent to the boundary $\partial D$ where $L$ coincides with $L_{0}$ and which separate regions of different colors. 
The graph $G(L)$ carries the following additional information: to each vertex $v \in V$ we associate a weight $w(v) \in(0,1 / 2)$ which equals the area of the connected component corresponding to $v$. Next, we introduce a linear ordering of the edges of $G(L)$ which corresponds to their order as segments of $L_{0}$ (going from left to right).
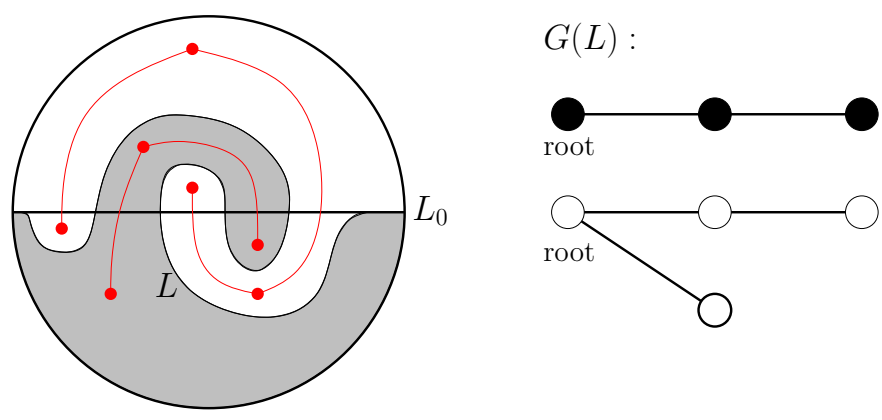

FiguRE 1. Graph construction

We have the following properties of $G=G(L)$ :

- $G$ is a union of two trees (one for the black vertices and another for the white ones). This follows from the fact that the set of vertices of the same color is connected in $G$ (the union of black / white regions is a half-disk), and it does not contain loops (otherwise the graph of the opposite color will become disconnected - each loop will bound a connected component).

The union of the two trees is not connected in $G$ since each edge connects vertices of the same color.

- Edges that connect white / black regions correspond to alternating segments on $L_{0}$. Therefore, the numbers of black and white vertices are either equal or differ by one.

- The sum of the weights of all white/black/northern/southern regions is equal to $1 / 2=\operatorname{Area}(D, \omega) / 2$.

- There are two distinguished vertices (a single black and a single white one) which correspond to the regions adjacent to the boundary $\partial D$, see Figure 1. These two vertices play special role because points near the boundary are stationary under compactly supported diffeomorphisms. We will call these vertices the roots of the corresponding trees.

- The total number of vertices is

$$
\# V(G)=\#\left(L_{0} \cap L\right)+3
$$

where the counting for \# $\left(L_{0} \cap L\right)$ goes only over the transverse intersections. 
The graph $G(L)$ is invariant under the action of Hamiltonians from $S$, therefore $G(L)$ depends only on the equivalence class $[L] \in \hat{\mathcal{E}}$. The following claim states that this correspondence $[L] \rightarrow G(L)$ is one-toone.

Proposition 5. Let $L_{1}, L_{2}$ be two diameters with isomorphic graphs, such that the graph isomorphism $g: G\left(L_{1}\right) \rightarrow G\left(L_{2}\right)$ preserves also weights, colors and ordering of the edges along $L_{0}$. Then there exists $\phi \in S$ such that $\phi\left(L_{1}\right)=L_{2}$.

Proof. Let $L_{1}, L_{2}$ be such that $G\left(L_{1}\right) \simeq G\left(L_{2}\right)$. Correspondence of the orderings of edges $E\left(L_{1}\right), E\left(L_{2}\right)$ implies that the intersection points of $L_{i} \cap L_{0}$ appear in the same order. We apply a deformation $\phi \in S$ supported in a neighbourhood of $L_{0}$ to ensure $L_{1} \cap U=L_{2} \cap U$ for some neighbourhood $U$ of $L_{0}$. Then, as corresponding regions of $D \backslash\left\{L_{i} \cup L_{0}\right\}$, $i=1,2$ have the same areas, there are no obstructions to deform each one into another by a Hamiltonian supported in $D \backslash L_{0}$. Applying these deformations consequently to each of the regions we eventually deform $L_{1}$ to $L_{2}$.

Remark. Not any pair of trees may come from this construction - there are topological/combinatorial restrictions on graphs which correspond to diameters in $D$.

We return to the proof of Theorem 2. Applying an appropriate Hamiltonian diffeomorphism to both $L, L^{\prime}$ we may assume that $L=$ $L_{0}$. In what follows we describe a way to construct a Hamiltonian flow which deforms $L^{\prime}$ to $L_{0}$ and whose Hofer length is bounded by $\frac{1}{8} \cdot \# L \cap L^{\prime}+c$, with $c \leq 1$.

Remark. The constant $\frac{1}{8}$ might not be sharp, however the previous section gives a lower bound of $\frac{1}{16}$ for the actual sharp constant.

Lemma 6. Let $\gamma_{1}, \gamma_{2}$ be two smooth embedded curves in $D$ which intersect transversely in two points. Denote by $A$ the closed domain bounded by $\gamma_{1} \cup \gamma_{2}$, and denote by a the area of $A$. Let $\Gamma$ be a smooth embedded curve which connects two boundary points $p_{1}, p_{2} \in \partial A\left(p_{1} \in \gamma_{1}\right.$, $\left.p_{2} \in \gamma_{2}\right)$ and does not touch $\gamma_{1} \cup \gamma_{2}$ except for the endpoints. Let $\gamma_{3}, \gamma_{4}$ be two curves in $D \backslash A$ which intersect $\Gamma$. Then for any small $\varepsilon>0$ there exists a Hamiltonian diffeomorphism $\phi_{\varepsilon}$ of norm at most $a+\varepsilon$, supported in a small neighborhood of $A \cup \Gamma$ such that $\phi_{\varepsilon}\left(\gamma_{2}\right) \cap \gamma_{1}=$ $\phi_{\varepsilon}\left(\gamma_{2}\right) \cap \gamma_{4}=\phi_{\varepsilon}\left(\gamma_{3}\right) \cap \gamma_{1}=\emptyset$.

Proof. Applying a symplectomorphism, if necessary, we may approximate $A$ by a rectangle. Consider the flow generated by a Hamiltonian 
$H_{\varepsilon}$ as shown in Figure 2. $H_{\varepsilon}$ is zero outside the outer rectangle, equals $a+\varepsilon$ in the inner part, linearly increases from $\frac{\varepsilon}{3}$ to $a+\frac{2 \varepsilon}{3}$ on $A$ and is smooth in the remaining region. It is easy to see, that for a small $\varepsilon$ and an appropriate choice of smoothing, the time-1 map of this flow gives the desired Hamiltonian diffeomorphism.
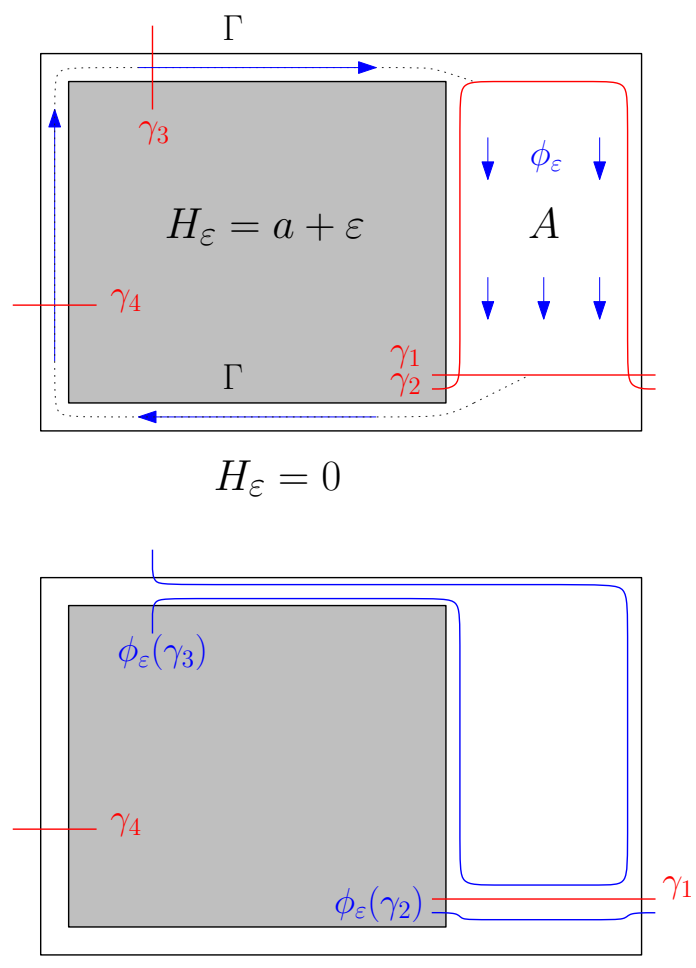

FiguRe 2.

Lemma 7. Let $G=G(L)$ be the graph corresponding to a diameter $L \subset D$. Let $v_{1} \in V(G)$ be a leaf different from the root and not adjacent to it, denote $a=w\left(v_{1}\right), v_{2}$ be a vertex in $G$ with graph distance $d_{G}\left(v_{1}, v_{2}\right)=2$. Then for arbitrary $\varepsilon>0$ there exists a Hamiltonian diffeomorphism $\phi$ with $\|\phi\|<a+\varepsilon$ which transfers weight a from $v_{1}$ to $v_{2}$ and removes $v_{1}$ from the graph without introducing new intersection points. (Such removal will result in merging of two vertices in the tree of the opposite color.) Application of such $\phi$ does not change areas of other regions by more than $\varepsilon$.

Proof. Without loss of generality we may assume that both $v_{1}, v_{2}$ are black northern vertices. Let $U$ be a neighbourhood of $v_{1}$ in $D$, denote 
$\gamma_{1}=L_{0} \cap U, \gamma_{2}=L \cap U$. The statement of the lemma implies that $\gamma_{2}$ is transverse to $\gamma_{1}$ in intersection points. Denote $\gamma_{3}=L \backslash \gamma_{2}, \gamma_{4}=L_{0} \backslash \gamma_{1}$. Let $v \in V(G)$ be the common neighbour of $v_{1}, v_{2}$. Pick a curve $\Gamma$ which starts from $\gamma_{1}$, intersects $L_{0}$ going through $v$ to $v_{2}$ along the black domain, and finally arrives to $\gamma_{2}$ without intersecting neither $\gamma_{2}$ nor $L_{0}$ anymore (see Figure 3).

The lemma follows by application of Lemma 6. The statement about $\gamma_{3}, \gamma_{4}$ ensures that $\phi$ does not create additional intersection points between $L_{0}$ and $L$.
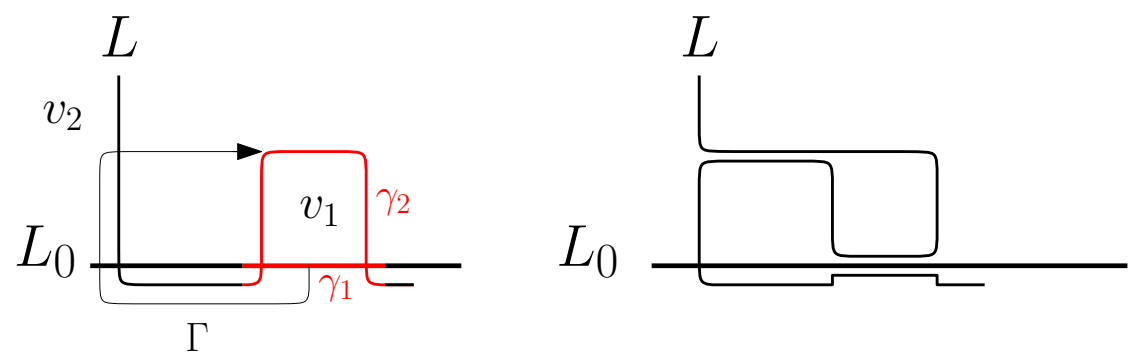

Figure 3. Leaf deletion

Proof of Theorem 2. Let $L^{\prime}$ be a diameter transverse to $L_{0}$. Denote by $T$ the black tree of $G\left(L^{\prime}\right)$. Ler $r$ be the root vertex of $T$. Denote by $n=\# L^{\prime} \cap L_{0}$ the number of transverse intersection points. Note that $\operatorname{depth}(T)$ is bounded by $\# E(T) \leq n / 2+1$.

We pass in turn over vertices of $T$, each time selecting a leaf, wiping it out the tree and transferring its weight 2 steps in direction of $r$ with the help of Lemma 7. In the end we remain with all the weight concentrated at the root and its neighbours, with all other vertices having disappeared from the graph. Weight of each vertex $v \in V(T)$ is involved in at most $\frac{d_{T}(v, r)}{2}$ operations of this type, therefore the total cost of these transfers is bounded by

$$
\sum\left\|\phi_{j}\right\| \leq \sum_{v_{i} \in T}\left(w\left(v_{i}\right)+\varepsilon\right) \cdot \frac{d_{T}\left(r, v_{i}\right)}{2}<\frac{1}{2} \cdot \frac{\operatorname{depth}(T)}{2}<\frac{n}{8}+\frac{1}{2} .
$$

It remains to transfer the weights from the neighbours of $r$ to the root. This operation can be achieved by a cost of $\sum_{v \neq r} w(v)+\varepsilon<1 / 2$. This is so because now the graph corresponds to a diameter which has a "simple" intersection pattern with $L_{0}$, as depicted in Figure 4 . 


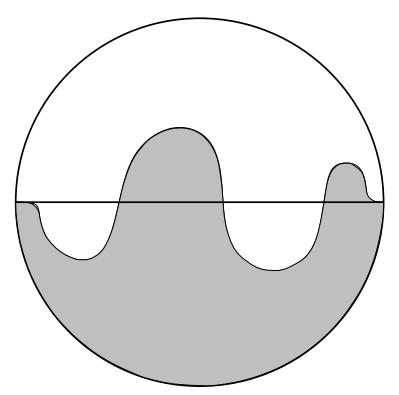

FiguRE 4.

\section{Generalizations}

One may try to extend the results of this paper to other surfaces. For compact surfaces $\Sigma$ of positive genus the problem is quite well understood. For example, using the argument introduced in [L-MD] one may prove that a closed embedded smooth curve $\gamma \subset \Sigma$ with nontrivial $[\gamma] \in H_{1}(\Sigma ; \mathbb{Z})$ can be pushed arbitrarily far from itself (in Hofer metric) using Hamiltonian isotopies.

In this section we state (without proof) few results for the cylinder $\Gamma=S^{1} \times(-1,1)$ and the sphere $S^{2}$ (both equipped with their standard symplectic structures).

We define equator as an image of a simple smooth closed curve which divides the manifold into two components of equal area. In the case of $\Gamma$ we add the requirement that the equator generates $H_{1}(\Gamma ; \mathbb{Z})$. An equivalent definition is that an equator is an image of the standard equator $L_{0}\left(L_{0}=\{z=0\}\right.$ for $S^{2}, L_{0}=S^{1} \times\{0\}$ for $\left.\Gamma\right)$ by a compactly supported Hamiltonian diffeomorphism.

In the case of $\Gamma$ both Theorem 1 and Theorem 2 remain true (with a different lower bound for the constant $K$ ). The proof is similar to that presented above. See [Kh-Thesis].

However, the case of $S^{2}$ is different. We do not know whether the space of equators $\mathcal{E}$ has finite or infinite Hofer diameter. The argument used in the proof of Theorem 1 fails as it is not known whether there exist two different homogeneous Calabi quasimorphism on $S^{2}$ (there exists at least one, see [E-P]). The question about finiteness of the diameter is closely related to the question of existance of another Calabi quasimorphism. For $S^{2}$ one may also show that $d\left(\phi^{t}\left(L_{0}\right), L_{0}\right) \leq c(\phi)$ (the constant is independent of $t$ ) for any family $\phi^{t} \in \operatorname{Ham}\left(S^{2}\right)$ generated by an autonomous flow. Therefore $L_{0}$ cannot be pushed infinitely 
far from itself using 1-parametric family of diffeomorphisms generated by any autonomous $F: S^{2} \rightarrow \mathbb{R}$.

A version of Theorem 2 remains true. Actually, using the fact that for $S^{2}$ the equator graphs do not have distinguished roots which contain stationary points, we can improve the linear estimate to a logarithmic one:

Theorem 8. (See Kh-Thesis) Let $L, L^{\prime}$ be two equators in $S^{2}$ with transverse intersections. Then for any $\varepsilon>0$,

$$
d\left(L, L^{\prime}\right) \leq \log _{9 / 4-\varepsilon}\left(\# L \cap L^{\prime}\right)+c(\varepsilon) .
$$

where $c(\varepsilon)$ is a constant which depends on $\varepsilon$ but not on the equators $L, L^{\prime}$.

\section{REFERENCES}

[Ch] Y. Chekanov, Invariant Finsler metrics on the space of Lagrangian embeddings, Math. Z., 234 (2000), 605-619.

[E-P] M. Entov, L. Polterovich, Calabi quasimorphisms and quantum homology, IMRN, no. 30, (2003), 1635-1676.

[Kh-Thesis] M. Khanevsky, PhD thesis, In preparation.

[L-MD] F. Lalonde, D. McDuff, Hofer's $L^{\infty}$-geometry: energy and stability of Hamiltonian flows, part II, Invent. Math. 122 (1995), 3569

Michael Khanevsky, School of Mathematical Sciences, Tel-Aviv UniVersity, Ramat-Aviv, Tel-Aviv 69978, IsRael

E-mail address: khanev@post.tau.ac.il 